\title{
Sleep-Disordered Breathing Is Independently Associated With Increased Aggressiveness of Cutaneous Melanoma
}

\section{A Multicenter Observational Study in 443 Patients}

Q23 Q1 M. A. Martinez-Garcia, MD; F. Campos-Rodriguez, MD; E. Nagore, MD; A. Martorell, MD; J. L. Rodriguez-Peralto, MD;

E. Riveiro-Falkenbach, MD; L. Hernandez, MD; J. Bañuls, MD; E. Arias, MD; P. Ortiz, MD; V. Cabriada, MD;

J. Gardeazabal, MD; J. M. Montserrat, MD; C. Carreres, MD; J. Corral, MD; J. F. Masa; J. Gomez de Terreros, MD;

J. Abad, MD; A. Boada, MD; O. Mediano, MD; E. de Eusebio, MD; E. Chiner, MD; P. Landete, MD; M. Mayos, MD;

A. Fortuño, MD; F. Barbé, MD; M. Sánchez de la Torre, MD; A. Sanchez de la Torre, MD; I. Cano, MD; C. Gonzalez, MD;

A. Pérez-Gil, MD; T. Gómez-García, MD; D. Cullen, MD; M. Somoza, MD; M. Formigón, MD; F. Aizpuru, MD; C. Navarro, MD; Q2 Q3 M. J. Selma-Ferrer, MD; A. Garcia-Ortega; B. de Unamuno, MD; I. Almendros, MD; R. Farré, MD; and D. Gozal, MD, MBA

Q6 Q5

BACKGROUND: Sleep-disordered breathing (SDB) has been associated with a greater incidence and mortality of cancer, although such findings are inconsistent. However, no large studies are currently available to investigate this association in patients with a specific type of cancer. This study seeks to assess potential relationships between SDB severity and aggressiveness markers of cutaneous melanoma.

METHODS: Four hundred and forty-three patients with a diagnosis of melanoma underwent a sleep study within 6 months of diagnosis. General demographics were collected, along with melanoma characteristics and polygraphic parameters consisting of the apnea-hypopnea index (AHI) and indices of both continuous and intermittent night-time oxyhemoglobin desaturation (DI4\%). An exploration of independent relationships between SDB and various objective melanoma aggressiveness markers (Breslow index, presence of ulceration, presence of regression, mitotic index, stage of severity, damage to the sentinel lymph, and spreading of the melanoma) was performed.

RESULTS: Patients in the upper tertiles of AHI or DI4\% were 1.94 (95\% CI, 1.14-3.32; $P=.022)$ and 1.93 (95\% CI, 1.14-3.26; $P=.013$ ) times more likely, respectively, to present with aggressive melanoma (Breslow index $>1 \mathrm{~mm}$ ) than those in the lowest tertiles of these sleep attributes after adjustment for age, sex, tumor location, and BMI. This association was particularly prominent among patients $<56$ years of age with Breslow index $>2 \mathrm{~mm}$. The presence of the additional markers of aggressiveness was also associated with higher AHI and DI $4 \%$ values.

CONCLUSIONS: The severity of SDB was independently associated with greater aggressiveness of cutaneous melanoma, particularly among younger patients.

CHEST 2018;

ABBREVIATIONS: AHI = apnea-hypopnea index; $\mathrm{CM}=$ cutaneous melanoma; $\mathrm{DI}=$ desaturation index; $\mathrm{IQR}=$ interquartile range; $\mathrm{SDB}=$ sleep-disordered breathing

AFFILIATIONS: From the Respiratory Department (Drs MartinezGarcia, Navarro, and Selma-Ferrer, and Mr Garcia-Ortega), Hospital
Universitario y Politécnico la Fe, Valencia, Spain; Respiratory Department (Dr Campos-Rodriguez), Hospital Valme, Seville, Spain; Dermatology Department (Dr Nagore), Instituto Valenciano de Oncología, Valencia, Spain; Dermatology Department (Dr Martorell), Hospital de Manises, Valencia, Spain; Anatomic Pathology

(c) <2018>. This manuscript version is made available under the CC-BY-NC-ND 4.0 license http://creativecommons.org/licenses/ 
Both cancer and sleep-disordered breathing (SDB) are major and challenging public health problems. ${ }^{1,2}$

Cutaneous melanoma (CM) is one of the most aggressive neoplastic tumors. There has been an estimated annual increase of 3\% to 7\% from 1990 to 2015 in the incidence of CM among Caucasian men. ${ }^{3}$ Although some of the major risk factors have been clearly established, other risk factors remain unknown, particularly those factors that determine the aggressiveness profile of the tumor. ${ }^{4}$ SDB affects $9 \%$ to $38 \%$ of the general population, and is characterized by repetitive collapse of the airway during sleep, leading to intermittent hypoxemia and sleep fragmentation. ${ }^{5}$ These two major components of SDB have been related to a higher incidence of traffic accidents and to cardiovascular, neuropsychiatric and metabolic disease. ${ }^{6,7}$

Emerging evidence has suggested an association between the presence of SDB and higher cancer prevalence, incidence, and mortality. ${ }^{8-10}$ This finding has been supported by pathophysiologic and experimental animal studies that have confirmed the biologic plausibility of such putative associations. ${ }^{11,12}$ Other authors, however, have failed to identify any significant link between the two conditions. ${ }^{13,14}$ Among the multiple possible explanations for these discrepancies, methodologic limitations may have played a key role, particularly when considering that the majority of the studies were retrospective or used databases that were not specifically designed to analyze this association. ${ }^{15}$

We have attempted here to overcome the aforementioned limitations, and examined the relationship between well-established severity measures of SDB and clinical aggressiveness markers of CM in a large cohort of patients receiving diagnoses with this tumor.

\section{Methods}

\section{Study Design and Participants}

This was a cross-sectional study that included consecutive patients who received a diagnosis of $\mathrm{CM}^{16}$ at 29 Spanish hospitals. Exclusion criteria were as follows: melanoma of an unknown primary site, melanoma in mucosa or melanomas "in situ," pregnant women, and respiratory or cardiac insufficiency. Subjects with previous continuous positive airway pressure treatment were also excluded, since this therapy counteracts the intermittent hypoxia associated with SDB, thereby decreasing the potential association between SDB and our endpoint The study was approved by the ethics committees of all the hospitals, and all the patients gave their informed consent.

\section{Data Collection}

Every participant completed a standardized protocol including cardiovascular and respiratory history and self-reported symptoms (witnessed apneas, chronic snoring, presence of daytime hypersomnia, sleep duration, and presence of insomnia). The following data were
Department (Drs Rodriguez-Peralto and Riveiro-Falkenbach), Hospital Universitario 12 de Octubre, Madrid, Spain; Respiratory Department (Dr Hernandez) and Dermatology Department (Dr Bañuls), Hospital General Universitario de Alicante, Alicante, Spain; Respiratory Department (Dr Arias) and Dermatology Department (Dr Ortiz), Hospital Universitario 12 de Octubre, Madrid, Spain; Respiratory Department (Dr Cabriada) and Dermatology Department (Dr Gardeazabal), Hospital Universitario Cruces de Bilbao, Bilbao, Spain; Respiratory Department (Dr Montserrat) and Dermatology Department (Dr Carreres), Hospital Clinic-IDIBAPS, Barcelona, Spain; Respiratory Department (Drs Corral and de Terreros, and Mr Masa), Hospital Universitario San Pedro de Alcántara, Cáceres, Spain; Respiratory Department (Dr Abad) and Dermatology Department (Dr Boada), Hospital Universitario Germans Trials I Pujol, Barcelona, Spain; Respiratory Department (Dr Mediano) and Dermatology Department (Dr de Eusebio), Hospital Universitario General de Guadalajara, Guadalajara, Spain; Respiratory Department (Drs Chiner and Landete), Hospital Universitario San Juan de Alicante, Alicante, Spain; Respiratory Department (Drs Mayos and Fortuño), Hospital Universitario Santa Creu i Sant Pau, Barcelona, Spain; Respiratory Department (Drs Barbé, M. Sánchez de la Torre, and A. Sanchez de la Torre), IRBLleida, Lleida, Spain; Respiratory Department (Dr Cano) and Dermatology Department (Dr Gonzalez), Hospital Universitario de Getafe, Madrid, Spain; Dermatology Department (Dr Pérez-Gil), Hospital Valme, Seville, Spain; Respiratory Department (Dr GómezGarcía) and Dermatology Department (Dr Cullen), Fundación Jimenez Diaz, Madrid, Spain; Respiratory Department (Dr Somoza) and Dermatology Department (Dr Formigón), Consorcio Sanitario Terrassa, Barcelona, Spain; Biostatistical Service (Dr Aizpuru), BioAraba, Health Research Institute, OSI Araba University Hospital, Basque
Health Service, University of the Basque Country; Dermatology Department (Dr de Unamuno), Hospital Universitario y Politécnico la Fe, Valencia, Spain; Unitat de Biofísica i Bioenginyeria (Drs Almendros and Farré), Facultat de Medicina i Ciències de la Salut, Universitat de Barcelona, Barcelona, Spain, Centro de Investigación Biomédica en Red de Enfermedades Respiratorias, Madrid, Spain, and Institut d'Investigacions Biomèdiques August Pi i Sunyer, Barcelona, Spain; Department of Pediatrics (Dr Gozal), University of Chicago, Chicago, IL; and CIBERes (Drs Montserrat, Corral, de Terreros, Mediano, Mayos, Fortuño, Barbé, M. Sánchez de la Torre, A. Sanchez de la Torre, Gómez-García, Almendros, and Farré, and Mr Masa), CIBER de Enfermedades Respiratorias, Madrid, Spain.

Part of this article has been presented at the 2015 European Respiratory Society International Congress, September 26-30, 2015, Amsterdam, the Netherlands and the American Thoracic Society 2016 International Congress, May 13-18, 2016, San Francisco, CA.

FUNDING/SUPPORT: This study was supported by grants from the Fondo de Investigation Sanitaria [Grant PI16/01772], SEPAR, Red Respira, and Sociedad Valenciana de Neumología. This study has not received any funding or grants from pharmaceutical or other industrial corporations. E. R. is the recipient of a postdoctoral fellowship from the "Fundación Científica de la Asociación Española contra el Cáncer." Q7 Q8 CORRESPONDENCE TO: Miguel Ángel Martínez-García, MD, ${ }^{\text {Q22 }}$ Pneumology Department, Hospital Universitario and Politécnico La Fe, Bulevar Sur s/n, 46012 Valencia, Spain; e-mail: mianmarti- Q10 nezgarcia@gmail.com

Copyright (c) 2018 American College of Chest Physicians. Published by Elsevier Inc. All rights reserved.

DOI: https://doi.org/10.1016/j.chest.2018.07.015 
also recorded with respect to the melanoma: the histologic type (superficial spreading, nodular, lentigo maligna, acral lentiginous, or others/unspecified); tumoral thickness (Breslow index, in $\mathrm{mm}$ ); the histologic presence of ulceration, regression, and the mitotic index (number of mitoses per $\left.\mathrm{mm}^{2}\right)^{17}$; and tumoral stage in accordance with the 7th edition of the staging classification system of the American Joint Committee on Cancer. ${ }^{18}$ The tumor was subsequently categorized as localized, locoregional, or distant. Tumor location was dichotomized according to its location on the head/neck/trunk (value, 1) or on limbs/acral area (value, 0 ).

\section{Procedures}

Sleep Study: All the patients underwent respiratory polygraphy that was performed in accordance with national guidelines. ${ }^{19}$ This sleep study was performed in all cases before 6 months had elapsed from the diagnosis of melanoma. Respiratory polygraphy included continuous recording of oronasal flow and pressure, heart rate, thoracic and abdominal respiratory movements, and oxygen saturation. Polygraphic data were scored manually by skilled staff. Apnea was defined as an interruption of oronasal airflow for $>10 \mathrm{~s}$. Hypopnea was defined as a $30 \%$ to $90 \%$ reduction in oronasal airflow for $>10 \mathrm{~s}$, associated with an oxygen desaturation $\geq 3 \%$. The apnea-hypopnea index (AHI) was defined as the number of apneas plus hypopneas per hour of recording, while Tsat $90 \%$ was defined as the percentage of recording night-time with oxygen saturation $<90 \%$. The recording time, desaturation indices (DI) at $3 \%$, and the mean baseline nocturnal, baseline daytime, and minimum nocturnal saturation were also recorded.

Endpoints: The primary endpoint was the association between SDB severity and $\mathrm{CM}$ aggressiveness as measured by the Breslow index. We used a Breslow index $>1 \mathrm{~mm}$ as one of the thresholds to define aggressiveness, according to the American Cancer Association, but also explored other proposed cutoff points $(>2$ and $>4 \mathrm{~mm}){ }^{18}$ As secondary endpoints, we used other markers of melanoma aggressiveness, such as the presence of ulceration or regression, a mitotic index $\geq 5 / \mathrm{mm},{ }^{2}$ damage to the sentinel lymph, and the various stages of melanoma severity. ${ }^{18}$

\section{Statistical Analysis}

The sample size was calculated by assuming an average age of 50 years in the patients with melanoma, ${ }^{4}$ a distribution of SDB similar to that of the general population, ${ }^{5}$ and $50 \%$ of melanomas with Breslow $>1 \mathrm{~mm} .{ }^{20}$ Accepting an $\alpha$ error $<0.05$ and a $\beta$ error $<0.20$, and foreseeing recruitment losses of $10 \%$, the minimum number of patients needed was 430 .

The SPSS 20.0 statistical package (IBM) was used for all analyses. Data were expressed by using the mean/median and standard deviation/ interquartile range according to the distribution of the variables. Qualitative and dichotomous variables were expressed as absolute and relative values. Comparison between the two groups' baseline variables (Breslow index $>1 \mathrm{~mm}$ vs $\leq 1 \mathrm{~mm}$ ) was made by Student $t$ test or the Mann-Whitney test, according to the distribution of the quantitative variables, while the $\chi^{2}$ test was used to compare the two percentages. Factors independently related to the presence of a Breslow index $>1$ were determined by introducing into a multivariate logistical regression analysis those variables that, in the opinion of the researchers, could have clinical importance: age, sex, BMI, alcohol consumption, smoking, and tumor location. Both the $\mathrm{AHI}$ and DI4\% were introduced into each logistic regression analysis as tertiles, with the lower tertile being considered the reference group. In addition, both AHI and DI4\% were analyzed as continuous variables, and using the usual clinical cutoff in AHI values $(5,15$, and 30 events $/ \mathrm{h})$. Various subgroups of patients of clinical interest were also analyzed, based on age, sex, presence of obesity (BMI $\geq 30 \mathrm{~kg} / \mathrm{m}^{2}$ ), and presence of hypersomnia (Epworth Sleepiness Scale $\geq 10$ ). Finally, the association between these polygraphic variables (AHI and DI4\% in tertiles) and other markers of melanoma aggressiveness was evaluated by means of the odds ratio (OR) (95\% CI), considering the lower tertile of severity as the reference group. $P<.05$ was considered significant in every case.

\section{Results}

\section{Baseline Characteristics}

Of the 476 patients who were initially recruited from October 2012 to September 2015, 443 were finally included (Fig 1). The patients' mean age was $55.9 \pm 15.3$ years and $50.6 \%$ were male. The median Breslow index score was 0.85 (interquartile range [IQR], 0.49-1.80) $\mathrm{mm}$. The median time span between CM diagnosis and sleep recording was 82 (IQR, 49-120) days. Table 1 shows the patients' general and melanoma characteristics, while Table 2 shows the main results of the sleep studies and clinical variables related to sleep. The median AHI was 8.6 (IQR, 2.8-20.2) events/h ( $65 \%$ with an $\mathrm{AHI} \geq 5$, and $14.7 \%$ an $\mathrm{AHI} \geq 30$ ), while the median value of DI $4 \%$ was 4.84 desaturations/h (IQR, 1.1-13.3).

\section{Comparison of Baseline Characteristics Between Groups According to the Breslow Index}

The comparison of baseline characteristics in patients with a Breslow index of $\leq 1 \mathrm{~mm}$ vs $>1 \mathrm{~mm}$ is shown in
Table 3. Higher age, male sex, greater BMI, and melanoma location, and all the measured polygraphic variables, including the desaturation indices, were statistically significantly higher in the group with more aggressive melanoma. However, there were no significant differences between the two groups regarding sleep symptoms.

\section{Sleep-Disordered Breathing Is Associated With Melanoma Aggressiveness}

Table 4 shows the findings of the logistic regression analysis, including both the $\mathrm{AHI}$ and the DI $4 \%$ as tertiles of growing severity. Those patients in an upper AHI tertile (AHI $>15.6$ events/h) or DI $4 \%$ tertile $(>9.3$ desaturations/h) exhibited an approximately twofold greater probability of presenting a melanoma with greater aggressiveness (Breslow $>1 \mathrm{~mm}$ ) compared with the reference group (lower tertile, AHI $<4.5$ or DI $4 \%<2$ ) adjusted by age, sex, BMI, and location of the melanoma. Similarly, both age and location of the melanoma were also independent indicators of greater melanoma aggressiveness. Using AHI and DI4\% as 


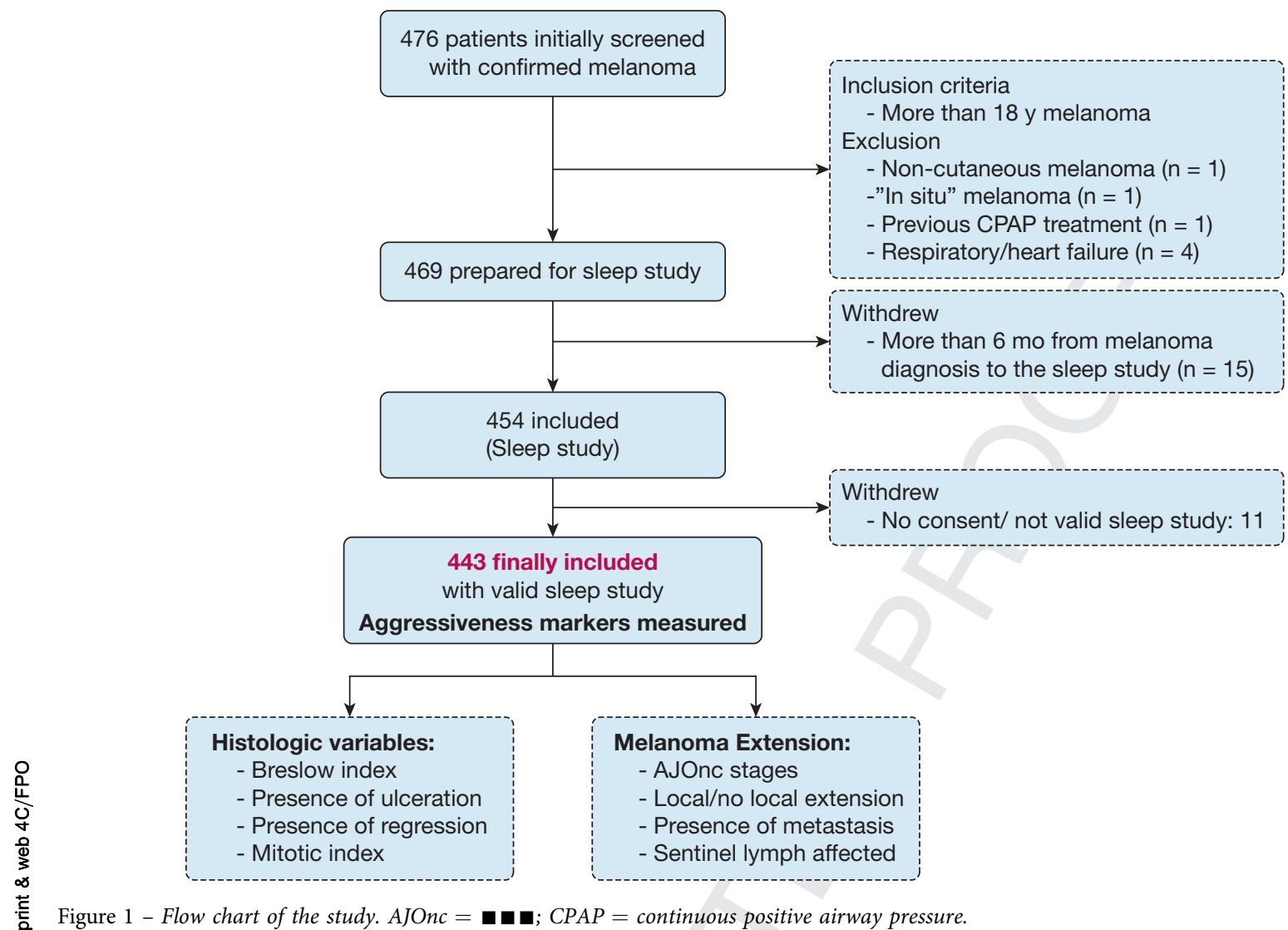

476 patients initially screened with confirmed melanoma

Figure 1 - Flow chart of the study. AJOnc

CPAP $=$ continuous positive airway pressure

continuous variables, and using standard clinical cutpoints for AHI, yielded similar patterns of association: both AHI (e-Table 1) and DI4\% (e-Table 2) remained significantly associated with greater melanoma aggressiveness.

\section{Analysis by Age Groups}

In patients $<56$ years, this association between sleep apnea and melanoma aggressiveness established by the Breslow index increased in robustness as the melanoma exhibited increasing Breslow index, while the opposite pattern emerged in patients $\geq 56$ years. Indeed, among older subjects the association between SDB measures (both the AHI and the DI4\%) and Breslow index was significant only in those patients with lower Breslow index values $(\leq 1)$ (Table 5$)$.

\section{Analysis by Other Clinical Relevant Groups}

The probability of having a Breslow index $>1$ in those patients with an AHI in the upper tertile compare with the reference group was higher in men $(n=224)$ than in women $(\mathrm{n}=219)$ and in nonobese $(\mathrm{n}=339)$ than in obese $(n=104)$ patients. Finally, regarding the presence of daytime hypersomnia $(n=71)$ or not $(\mathrm{n}=372)$, the ORs were not statistically significant in both cases. The findings were markedly similar when DI4\% tertiles were used as the SDB polygraphic variable (e-Table 3$)$.

\section{Relationship With Other Melanoma Aggressiveness Markers}

Additional melanoma aggressiveness markers were also significantly associated with the upper AHI and DI4\% tertiles (with ORs between 1.4 and 2.6 and between 2 and 2.55, compared with the lower tertiles, respectively). Only the presence of regression did not exhibit a statistically significant association with DI4\% (Fig 2).

\section{Severity-Dependent Effect Relationship}

Figure 3 shows the presence of a "severity-dependent effect," that is, the more advanced the stage of the $\mathrm{CM}$ lesion according to the classification of the American Joint Committee on Cancer ${ }^{18}$ the greater the values of both the AHI and the DI4\% in a quantitative analysis.

\section{Discussion}

To our knowledge, this study shows for the first time Q25 that there is a positive, independent association between 
TABLE 1 ] Patient and Cutaneous Melanoma Characteristics at Baseline

\begin{tabular}{|c|c|}
\hline Parameter & Value \\
\hline Patients, No. (\%) & $443(100)$ \\
\hline Male sex, No. (\%) & $224(50.6)$ \\
\hline Age, mean $\pm S D$, y & $55.98 \pm 15.3$ \\
\hline $\mathrm{BMI}$, mean $\pm \mathrm{SD}, \mathrm{kg} / \mathrm{m}^{2}$ & $27.3 \pm 4.5$ \\
\hline BMI $\geq 30$, No. (\%) & $104(23.5)$ \\
\hline Breslow index, ${ }^{a}$ thickness in $\mathrm{mm}$ & $0.85(0.49-1.80)$ \\
\hline Ulceration, No. (\%) & $71(16)$ \\
\hline Regression, No. (\%) & $103(23.3)$ \\
\hline \multicolumn{2}{|l|}{ Mitotic rate, mitoses $/ \mathrm{mm}^{2}$, No. (\%) } \\
\hline $0-1 / \mathrm{mm}^{2}$ & $278(64.5)$ \\
\hline$>5 / \mathrm{mm}^{2}$ & $63(14.2)$ \\
\hline \multicolumn{2}{|l|}{ Subtype, No. (\%) } \\
\hline Superficial spreading melanoma & $312(70.4)$ \\
\hline Nodular melanoma & $66(14.9)$ \\
\hline Lentigo maligna melanoma & $34(7.7)$ \\
\hline Acral lentiginous melanoma & $23(5.3)$ \\
\hline $\begin{array}{l}\text { Sentinel lymph node affected, } \\
\text { No. (\%) }\end{array}$ & $47(10.6)$ \\
\hline \multicolumn{2}{|l|}{ Anatomical site, No. (\%) } \\
\hline Head and neck & $64(14.4)$ \\
\hline Trunk & $178(40.2)$ \\
\hline Upper limb & $67(15.1)$ \\
\hline Lower limb & $113(25.5)$ \\
\hline Acral & $20(4.5)$ \\
\hline \multicolumn{2}{|l|}{ Stage, No. (\%) } \\
\hline Localized (I-II) & $387(87.4)$ \\
\hline Locoregional (III) & $46(10.4)$ \\
\hline Metastatic (IV) & $4(0.9)$ \\
\hline
\end{tabular}

${ }^{a}$ Breslow index is expressed as median (interquartile range).

the number and severity of SDB (measured as both AHI and DI4\%), and various commonly used aggressiveness markers of $\mathrm{CM}$ in a large series of patients. This association was more robust among younger patients in the context of higher Breslow values. Considering that SDB is a treatable disease, and although further studies are needed to demonstrate the role of SDB and its treatment in the evolution of $\mathrm{CM}$, our results emphasize the need to raise awareness among physicians treating patients with melanoma of the possibility of underlying SDB.

The melanoma model has been used in this study for several reasons: (1) an initial pilot study undertaken by our research group on 56 patients with CM found a significant association between the severity of SDB
TABLE 2 S Sleep Characteristics and Other Comorbidities at Baseline

\begin{tabular}{|c|c|}
\hline Parameter & Value \\
\hline Patients, No. (\%) & $443(100)$ \\
\hline Smoking, pack-years (if $>0)^{a}$ & $17(8-30)$ \\
\hline Current, No. (\%) & $82(18.5)$ \\
\hline Past, No. (\%) & $138(31.2)$ \\
\hline Never, No. (\%) & $221(49.9)$ \\
\hline Alcohol, g/d (if $>0)^{a}$ & $15(10-30)$ \\
\hline Never, No. (\%) & $363(81.9)$ \\
\hline Previous cancer, No. (\%) & $34(7.7)$ \\
\hline $\begin{array}{c}\text { Previous chronic respiratory/ } \\
\text { heart disease, No. (\%) }\end{array}$ & $33(7.4)$ \\
\hline \multicolumn{2}{|l|}{ Site of sleep study, No. (\%) } \\
\hline Hospital & $123(27.8)$ \\
\hline Home & $320(72.2)$ \\
\hline Sleep study time, $\mathrm{h}^{\mathrm{a}}$ & $7.2(6.6-8)$ \\
\hline $\begin{array}{l}\text { Chronic snoring (at least } 3 \mathrm{~d} / \mathrm{wk} \text { ), } \\
\text { No. }(\%)^{\mathrm{b}}\end{array}$ & $278(62.8)$ \\
\hline $\mathrm{d} / \mathrm{wk}$, mean $\pm S D$ & $3.7 \pm 2.96$ \\
\hline Witnessed apneas, No. $(\%)^{\mathrm{b}}$ & $88(19.9)$ \\
\hline Epworth test ${ }^{a, b}$ & $6(3-8)$ \\
\hline Epworth test $\geq 10$, No. (\%) & $71(16)$ \\
\hline Neck circumference, mean $\pm \mathrm{SD}, \mathrm{cm}$ & $37.7 \pm 4.5$ \\
\hline Sleep duration, mean $\pm S D$, $h^{b}$ & $7.4 \pm 1.27$ \\
\hline Less than 6 h, No. (\%) & $21(4.7)$ \\
\hline Between 6 and 8 h, No. (\%) & 354 (77.9) \\
\hline More than $8 \mathrm{~h}$, No. (\%) & $77(17.4)$ \\
\hline Insomnia, No. $(\%)^{\mathrm{b}}$ & $37(8.4)$ \\
\hline 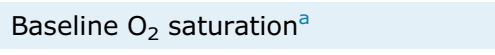 & $97(96-98)$ \\
\hline$A H I$, No. of events $/ h^{a}$ & $8.6(2.8-20.2)$ \\
\hline $\mathrm{AHI} \geq 5$, No. $(\%)$ & $288(65)$ \\
\hline $\mathrm{AHI} \geq 15$, No. $(\%)$ & $156(35.2)$ \\
\hline $\mathrm{AHI} \geq 30$, No. (\%) & $65(14.7)$ \\
\hline Central AHI, events $/ \mathrm{h}^{\mathrm{a}}$ & $0(0-0.6)$ \\
\hline DI4\%, No. of desaturations $/ \mathrm{h}^{\mathrm{a}}$ & $4.84(1.1-13.3)$ \\
\hline DI3\%, No. of desaturations $/ h^{a}$ & $9(2.8-21.6)$ \\
\hline Minimum $\mathrm{O}_{2}$ saturation ${ }^{a}$ & $85(80-89)$ \\
\hline Nocturnal average $\mathrm{O}_{2}$ saturation ${ }^{a}$ & $93(91-96)$ \\
\hline Tsat90\% ${ }^{a}$ & $0.65(0-5.25)$ \\
\hline
\end{tabular}

$\mathrm{AHI}=$ apnea-hypopnea index; DI4\% and DI3\% = desaturation index at $4 \%$ and $3 \%$, respectively; Tsat $90 \%=$ night time spent with oxygen saturation below $90 \%$.

asmoking, alcohol use, sleep study time, Epworth test, diurnal $\mathrm{O}_{2}$ saturation, $\mathrm{AHI}$, central AHI, DI4\%, DI3\%, nocturnal average $\mathrm{O}_{2}$ saturation, Q20 and $\mathrm{Tsat} 90 \%$ are expressed as median (interquartile range).

bSelf-reported variable.

and melanoma aggressiveness (patients from this pilot study were not included in the present study) ${ }^{21}$; (2) several authors have suggested that melanoma 
TABLE 3 ] Comparison of Sleep-Disordered Breathing Severity Measures in Groups With a Breslow Index $>1$ mm and $\leq 1 \mathrm{~mm}$

\begin{tabular}{|c|c|c|c|}
\hline Variable & Breslow Index $>1$ mm $(n=195)$ & Breslow Index $\leq 1 \mathrm{~mm}(\mathrm{n}=248)$ & $P$ Value \\
\hline Age, mean $\pm S D, y$ & $59.52 \pm 15.3$ & $53.2 \pm 14.8$ & .0001 \\
\hline Sex, male, No. (\%) & $109(55.9)$ & 115 (39.9) & .029 \\
\hline $\mathrm{BMI}$, mean $\pm \mathrm{SD}, \mathrm{kg} / \mathrm{m}^{2}$ & $27.9 \pm 4.5$ & $26.8 \pm 4.4$ & .015 \\
\hline Smoking, mean $\pm S D$, pack-years & $12.5 \pm 19.9$ & $9.3 \pm 14.8$ & .32 \\
\hline Alcohol, mean $\pm S D, g / d$ & $4 \pm 15$ & $4.2 \pm 12.6$ & .39 \\
\hline Melanoma site, No. (\%) & & & .035 \\
\hline Head/neck & $26(13.3)$ & $38(13.2)$ & \\
\hline Arms & $26(13.3)$ & $41(14.2)$ & \\
\hline Legs & $55(28.2)$ & $58(20.1)$ & \\
\hline Trunk & $72(36.9)$ & $106(36.8)$ & \\
\hline Acral & $15(7.7)$ & $5(1.7)$ & \\
\hline AHI, No. of events $/ h^{a}$ & $13(4.5-25)$ & $6.4(2.1-15.6)$ & .0001 \\
\hline $\mathrm{AHI} \geq 5$, No. $(\%)$ & $144(73.8)$ & $144(50)$ & .0001 \\
\hline $\mathrm{AHI} \geq 15$, No. $(\%)$ & $89(45.6)$ & $67(23.3)$ & .0001 \\
\hline $\mathrm{AHI} \geq 30$, No. $(\%)$ & 37 (19) & $28(9.7)$ & .017 \\
\hline DI3\%, No. of events $/ h^{a}$ & $12.9(3.9-24.6)$ & $7.1(2.3-17.6)$ & .001 \\
\hline DI4\%, No. of events $/ h^{a}$ & $7(2.2-15.5)$ & $3.4(0.9-10.7)$ & .0001 \\
\hline Tsat90\% ${ }^{a}$ & $1.4(0.1-5.9)$ & $0.3(0-4.3)$ & .005 \\
\hline${\text { Baseline } \mathrm{O}_{2} \text { saturation }}^{a}$ & $96(95-97)$ & $96(95-97)$ & .22 \\
\hline Minimum $\mathrm{O}_{2}$ saturation ${ }^{a}$ & $85(78-88)$ & $86(81-89.3)$ & .0001 \\
\hline Sleep study time, $\mathrm{h}^{\mathrm{a}}$ & $7.2(6.5-8)$ & $7.2(6.7-8)$ & .84 \\
\hline Sleep duration, h/d & $7.7(1.1)$ & $7.3(1.3)$ & .009 \\
\hline \multicolumn{4}{|l|}{ Sleep-related symptoms } \\
\hline Chronic snoring, No. (\%) & $123(63.1)$ & $155(53.8)$ & .46 \\
\hline Witnessed apneas, No. (\%) & $42(21.5)$ & $46(16)$ & .73 \\
\hline $\mathrm{ESS}^{\mathrm{a}}$ & $6(3-8.3)$ & $6(3-8)$ & .91 \\
\hline
\end{tabular}

ESS $=$ Epworth Sleepiness Scale. See Table 2 legend for expansion of other abbreviations.

${ }^{\mathrm{a}} \mathrm{AHI}$, DI3\%, DI4\%, Tsat90\%, baseline and minimum $\mathrm{O}_{2}$ saturations, sleep study time, and ESS values are expressed as median (interquartile range).

could be one of the neoplastic conditions most likely to be associated with $\mathrm{SDB}^{22}$; (3) studies on animals injected with melanoma cells and subjected to intermittent hypoxemia showed faster growth of the tumor when compared with mice exposed to normoxic conditions ${ }^{11}$; and, finally, (4) melanoma is a prevalent and clinically significant cancer with well-documented objective markers of aggressiveness, especially the Breslow index. ${ }^{4,16}$ Since there is no natural cutoff point in the Breslow index that clearly defines melanoma aggressiveness, we finally used a cutoff point of $1 \mathrm{~mm}$ (one of the points defined by the current classification of the American Joint Committee on Cancer $^{18}$ ), due to the distribution of the Breslow index in our series (median value, $0.85 \mathrm{~mm}$ ).
The relationship between SDB and cancer remains controversial. Nieto et $\mathrm{al}^{10}$ observed in a study of the Wisconsin cohort that patients with an $\mathrm{AHI} \geq 30$ were 4.8 times more likely to die of cancer than those with an $\mathrm{AHI}<5$, and almost twice as likely if a higher nocturnal desaturation index was chosen as a marker of SDB severity. Similar results were reported by Marshall et $\mathrm{al}^{23}$ in 393 patients, but both these studies were carried out on individuals of the general population or on patients with sleep apnea, rather than in patients with cancer. Other authors, however, have had different findings. Gozal et $\mathrm{al}^{22}$ in an epidemiologic study of case and control subjects, using a health insurance database with more than 1.7 million patients with sleep apnea, found that this type of SDB was related to a higher incidence of some tumors (including melanoma) when compared with a 
TABLE 4 ] Variables Independently Associated With the Presence of a Cutaneous Melanoma Tumor With Greater Aggressiveness (Breslow Index $>1 \mathrm{~mm}$ ) in a Model Including AHI Tertiles, and a Model Including DI4\% Tertiles

\begin{tabular}{|c|c|c|c|c|}
\hline & Nonadjusted OR (95\% CI) & $P$ Value & Fully adjusted OR (95\% CI) & $P$ Value \\
\hline \multicolumn{5}{|c|}{ Model Including AHI (but not DI4\%) } \\
\hline \multicolumn{5}{|l|}{ AHI, events/h } \\
\hline \multicolumn{5}{|l|}{ Tertile } \\
\hline $0-4.5$ & 1 & $\ldots$ & 1 & $\ldots$ \\
\hline $4.6-15.6$ & $1.41(0.88-2.27)$ & .16 & $1.18(0.71-1.94)$ & .52 \\
\hline$>15.6$ & $2.76(1.72-4.42)$ & .0001 & $1.96(1.14-3.36)$ & .014 \\
\hline Age, y & $1.03(1.02-1.04)$ & .0001 & $1.02(1.01-1.03)$ & .017 \\
\hline Melanoma location & $1.36(0.93-1.98)$ & .11 & $1.57(1.01-2.32)$ & .049 \\
\hline BMI & $1.05(1.01-1.10)$ & .016 & $1.02(0.97-1.07)$ & .8 \\
\hline Sex & $1.47(1.01-2.14)$ & .096 & $1.33(0.91-2.15)$ & .27 \\
\hline Alcohol consumption, $\mathrm{g} / \mathrm{d}$ & $1.01(0.99-1.02)$ & .21 & $1.00(0.99-1.02)$ & .45 \\
\hline Smoking, pack-years & $1.01(0.99-1.02)$ & .25 & $0.99(0.99-1.01)$ & .93 \\
\hline \multicolumn{5}{|c|}{ Model Including DI4\% (but not AHI) } \\
\hline \multicolumn{5}{|l|}{ DI4\%, desaturations/h } \\
\hline \multicolumn{5}{|l|}{ Tertile } \\
\hline $0-2$ & 1 & $\ldots$ & 1 & $\ldots$ \\
\hline $2.1-9.3$ & $1.49(0.92-2.41)$ & .11 & $1.22(0.73-2.02)$ & .45 \\
\hline$>9.3$ & $2.71(1.69-4.34)$ & .0001 & $1.95(1.15-3.30)$ & .013 \\
\hline Age, y & $1.03(1.02-1.04)$ & .0001 & $1.02(1.01-1.04)$ & .01 \\
\hline Melanoma location & $1.36(0.93-1.98)$ & .11 & $1.43(1.01-2.17)$ & .047 \\
\hline BMI & $1.05(1.01-1.10)$ & .016 & $1.02(0.97-1.06)$ & .61 \\
\hline Sex & $1.47(1.01-2.14)$ & .096 & $1.21(0.77-1.90)$ & .41 \\
\hline Alcohol consumption, $\mathrm{g} / \mathrm{d}$ & $1.01(0.99-1.02)$ & .21 & $1.01(0.99-1.02)$ & .42 \\
\hline Smoking, pack-years & $1.01(0.99-1.02)$ & .25 & $1.00(0.99-1.01)$ & .96 \\
\hline
\end{tabular}

$\mathrm{OR}=$ odds ratio. See Table 2 legend for expansion of other abbreviations.

aFully adjusted odds ratio: age, sex (0, men; 1, women), BMI, melanoma location, alcohol consumption, smoking, and AHI/DI4\% tertiles. Melanoma location: variable dichotomized according to its location on the head/neck/trunk (value, 1) or on limbs/acral area (value, 0).

similar number of control subjects, but also reported a lower incidence of other malignant tumors, with no compelling evidence to suggest that any of the cancer

Q26 types examined was more aggressive. To our knowledge, our study is the first to assess a sufficient sample size of patients with a specific type of cancer (in this case, CM) to demonstrate a positive and independent association between SDB severity, measured by both the AHI and the ID4\%, and melanoma aggressiveness, objectively quantified using various well-established markers, and more particularly the Breslow index.

Among the most salient findings were the dependence of the association between SDB and the markers of melanoma aggressiveness on both age and the actual indicators of tumor aggressiveness. Accordingly, in the group of younger patients $(<55$ years old $)$, the SDBmelanoma relationship was significant only in more aggressive melanomas (ie, values $>2 \mathrm{~mm}$ on the Breslow index). In contrast, in the older patients, a significant association of melanoma aggressiveness with SDB measures was found only in those melanomas when Breslow indices were $<1 \mathrm{~mm}$ at the time of CM diagnosis. This intriguing age-dependent bifurcation in the association with SDB was apparent despite the fact that age per se was a variable independently and linearly associated with greater aggressiveness of the CM lesion. The potential explanation for this phenomenon is unclear. However, other studies investigating the association between cancer and sleep apnea in humans ${ }^{8,9}$ and between intermittent hypoxemia and cancer in animals ${ }^{11}$ have 
TABLE 5 ] Association Between Aggressiveness of Cutaneous Melanoma at Diagnosis and Severity of SDB as Measured by AHI and DI4\%, According to the Breslow Index Cutoff Points Established by the 2010 American Joint Committee on Cancer and According to the Age of the Patients ${ }^{a}$

\begin{tabular}{|c|c|}
\hline $\begin{array}{l}\text { AHI, events/h } \\
1^{0} \text { tertile }(0-4.5) \text { vs } 3^{\circ} \text { tertile }(>15.6)\end{array}$ & OR $(95 \% \mathrm{CI})$ \\
\hline Breslow $>1 \mathrm{~mm}(\mathrm{n}=195)$ & $\begin{array}{l}<56 \text { y }(\mathrm{n}=215), 1.32(0.85-3.01 ; P=.44) \\
\geq 56 \text { y }(\mathrm{n}=228), 3.18(1.85-7.23 ; P=.02)\end{array}$ \\
\hline Breslow > 2 mm $(n=99)$ & $\begin{array}{l}<56 \text { y }(\mathrm{n}=215), 2.83(1.12-7.33 ; P=.03) \\
\geq 56 \text { y }(\mathrm{n}=228), 1.53(0.25-3.66 ; P=.58)\end{array}$ \\
\hline Breslow $>4 \mathrm{~mm}(\mathrm{n}=39)$ & $\begin{array}{l}<56 \text { y }(n=215), 8.01(1.35-17.55 ; P=.04) \\
\geq 56 \text { y }(n=228), 0.60(0.19-1.55 ; P=.49)\end{array}$ \\
\hline $\begin{array}{l}\text { DI4\%, desaturations/h } \\
1^{\circ} \text { tertile }(0-2) \text { vs } 3^{\circ} \text { tertile }(>9.3)\end{array}$ & OR $(95 \% \mathrm{CI})$ \\
\hline Breslow > $1 \mathrm{~mm}(n=195)$ & $\begin{array}{l}<56 \text { y }(\mathrm{n}=215), 1.91(0.96-4.76 ; P=.11) \\
\geq 56 \text { y }(\mathrm{n}=228), 2.14(0.97-5.11, P=.09)\end{array}$ \\
\hline Breslow > 2 mm $(n=99)$ & $\begin{array}{l}<56 \text { y }(n=215), 3.87(1.33-11.56 ; P=.03) \\
\geq 56 \text { y }(n=228), 1.35(0.77-3.01 ; P=.34)\end{array}$ \\
\hline Breslow $>4 \mathrm{~mm}(\mathrm{n}=39)$ & $\begin{array}{l}<56 \text { y }(n=215), 7.94(1.78-16.45 ; P=.03) \\
\geq 56 \text { y }(n=228), 0.60(0.18-1.67 ; P=.33)\end{array}$ \\
\hline
\end{tabular}

SDB $=$ sleep-disordered breathing. See Table 2 and 3 legends for expansion of other abbreviations.

${ }^{a}$ Cutoff point at $56 y$.

revealed similar patterns. This may reflect interactions between the highly variable immune deregulation that traditionally occurs with aging and the now wellestablished alterations in immune responses elicited by sleep apnea. ${ }^{24}$ It is also possible that the reduced tumoral immunovigilance resulting from the aging

\section{A}

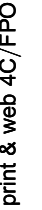

process may hinder any manifestation of the overall effects of sleep apnea, or that these effects may have disappeared altogether due to the inability of sleep apnea to elicit any changes in the susceptible immune pathways of the elderly. It would therefore be anticipated that younger patients with more robust

B

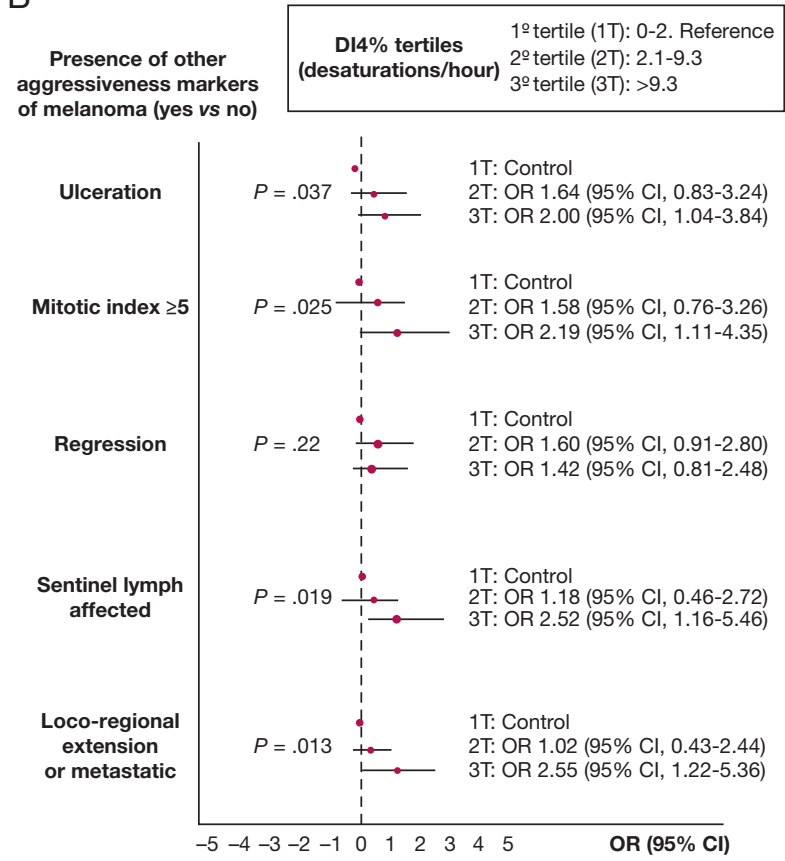

Figure $2-A$ and B, Relationship between (A) AHI and (B) DI4\% and other commonly used clinical markers of tumor aggressiveness in cutaneous melanoma. P values refer to upper tertile vs lower tertile. AHI = apnea-hypopnea index; DI4\% = desaturation index at $4 \%$. 

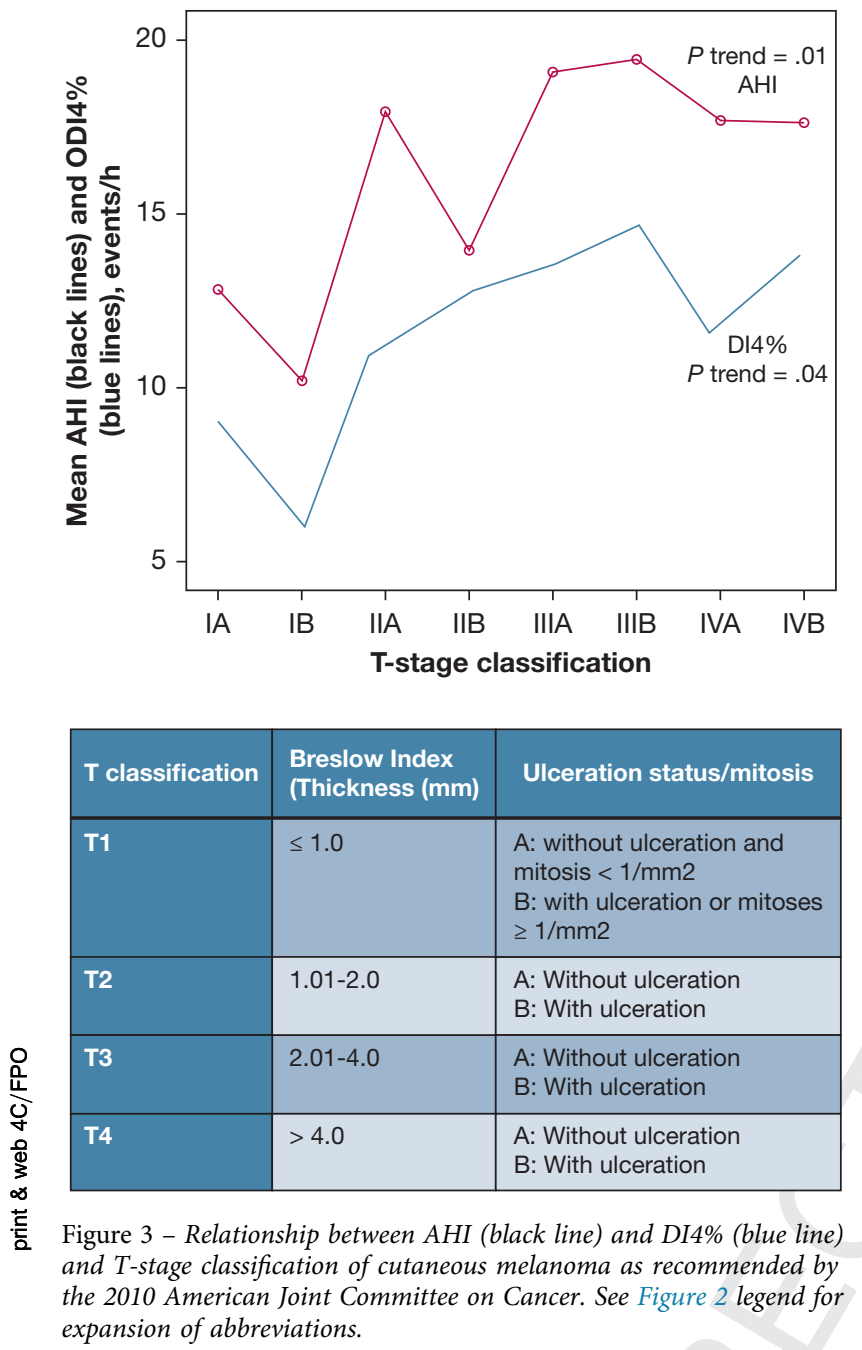

tumoral immunovigilance, and thus a more favorable prognosis of CM, would be more likely to manifest detectable adverse effects when concomitant sleep apnea is present. ${ }^{25-28}$

Beyond the main strength of the study related to the relatively large cohort size that enables appropriate gauging of the potential effects of SDB on a single neoplasia, there are some limitations that are worthy of mention. The absence of full overnight polysomnography as a diagnostic method precludes assessment on the role of sleep fragmentation or duration, both of which have been suggested as being participatory in the possible link between sleep apnea and cancer. ${ }^{29-31}$ Our decision to use respiratory polygraphy aimed to ensure a more representative participation by patients from 29 hospitals from all over Spain, since several of the participating centers had no access to polysomnography.

In summary, current findings support the presence of a positive, independent association between sleep apnea and various commonly used clinical markers of melanoma aggressiveness. Such findings were particularly apparent in younger patients with more aggressive melanomas. Future prospective studies are needed to confirm whether the presence and treatment of SDB and its evolution over time are also associated with poor melanoma outcomes, including death, and the pathophysiologic mechanisms underlying this association. 


\section{Acknowledgments}

Author contributions: M. A. M.-G. is the guarantor of the content of the manuscript, including data and analysis. Conception and design: M. A. M.-G., F. C.-R., E. N., A. M., J. L. R.-P., E. R.-F., I. A., R. F., D. G. Acquisition and interpretation of data: M. A. M.-G., F. C.R., E. N., A. M., L. H., J. B., E. A., P. O., V. C., J. G., J. M. M., C. C., J. C., J. G. d. T., J. A., A. B., O. M., E. d. E., E. C., P. L., M. M., A. F., F. B., M. S. d. 1. T., A. S. d. 1. T., I. C., C. G., A. P.-G., T. G.-G., D. C., M. S., M. F., C. N., M. J. S.-F., B. d. U. Drafting of manuscript and critical revision of major intellectual content: All authors. Final approval of the version to be published: All authors.

Financial/nonfinancial disclosure: None declared.

Role of sponsors: The sponsor had no role in the design of the study, the collection and analysis of the data, or the preparation of the manuscript.

Spanish Sleep Network: Isabel Betlloch, MD, Dermatology Department, Hospital General Universitario de Alicante; Manuel Moragon, MD, Dermatology Department, Hospital San Juan de Alicante; Esther Pastor, MD, Pneumology Service, Hospital San Juan de Alicante; Verónica Velasco, MD,

Dermatology Department, Hospital de Cruces de Bilbao; Amaia Urrutia, MD, Respiratory Department, Hospital de Cruces de Bilbao; Cristian Perna, MD, Anatomic Pathology Department, Hospital de Guadalajara; Zully Vásquez, MD, Respiratory Department, Hospital de Getafe; Jose Antonio Aramburu, MD, Anatomic Pathology Department, Hospital de Getafe; Aida Muñoz, MD, Respiratory Department, Hospital Germans Trials i Pujol; M. Angeles Gonzalez, MD, Anatomic Pathology Department, Hospital San Pedro de Alcántara, Cáceres; Joan Dalmau, MD, Dermatology Department, Hospital de Santa Creu i Sant Pau, Barcelona; Rosa M. Marti, MD, Dermatology Department, Hospital Arnau de Vilanova, Lleida; Ana Mozos, MD, Anatomic Pathology Department, Hospital de Santa Creu i Sant Pau, Barcelona; Erika Miranda, MD, Biostatistical Service, BioAraba, Health Research Institute, OSI Araba University Hospital, Basque Health Service; Maider Mateos, MD, Biostatistical Service, BioAraba, Health Research Institute, OSI Araba University Hospital, Basque Health Service; Paula Rodriguez, MD, Respiratory Department, Fundación Jiménez Diaz, Madrid; M. Fernanda Troncoso, MD, Respiratory Department, Fundación Jiménez Diaz, Madrid, CIBER de Enfermedades Respiratorias, Madrid; Monica de la Peña, MD, Respiratory Department, Hospital Son Espases, Palma de Mallorca; Luis Javier del Pozo, Dermatology Department, Hospital Son Espases, Palma de Mallorca; Mónica Gonzalez, MD, Respiratory Department, Hospital Marqués de Valdecilla, Santander; Pilar Manchado, MD, Dermatology Department, Hospital Clínico Universitario de Valladolid; Santiago Antonio Juarros, MD, Respiratory Department, Hospital Clínico
Universitario de Valladolid; Julian Caballero, MD, Respiratory Department, Hospital Morales Meseguer, MD, Murcia; Eduardo Alcaraz, MD, Anatomic Pathology Department, Hospital Morales Meseguer, Murcia; Andrea Crespo, MD, Respiratory Department, Hospital Rio Hortega, Valladolid; Jesús Martín, Anatomic Pathology Department, Hospital Rio Hortega, Valladolid; Lorena Comeche, MD, Respiratory Department, Hospital Universitario Quirónsalud, Madrid; Martina Alés, Dermatology Department, Hospital Universitario Quirónsalud, Madrid; Monica Llombart, MD, Respiratory Department, Hospital Marina Baixa, Alicante; María Pérez-Crespo, MD, Dermatology Department, Hospital Marina Baixa, Alicante; Bienvenido Barreiro, MD, Respiratory Department, Hospital Mutua Terrassa, Barcelona; Neus Curco, MD, Dermatology Department, Hospital Mutua Terrassa, Barcelona; Olga Cantalejo, MD, Hospital Universitario Marqués de Valdecilla, Santander; Nuria Reyes-Nuñez, MD, Respiratory Department, Hospital de Valme, Seville; Antonio Cruz-Medina, MD, Respiratory Department, Hospital Valme, Seville; Raquel Catalá, MD, Respiratory Department, IISPV, Universitat Rovira i Virgili, Reus; Antonio Azón, MD, Dermatology Department, Universitat Rovira i Virgili, Reus; Nuria Grau, Respiratory Department, Institut Hospital del Mar d'Investigacions Mêdiques, Barcelona; Sonia Segura, Dermatology Department, Institut Hospital del Mar d'Investigacions Mêdiques, Barcelona. All from Spain.

Additional information: The e-Tables can be found in the Supplemental Materials section of the online article.

\section{References}

1. Phillipson EA. Sleep apnea-a major public health problem. $N$ Engl J Med. 1993;328(17):1271-1273.

2. Maddams J, Utley M, Møller H. Projections of cancer prevalence in the United Kingdom 2010-2040. Br J Cancer. 2012;107(7):1195-1202.

3. Global Burden of Disease Cancer Collaboration. Global, regional, and national cancer incidence, mortality, years of life lost, years lived with disability, and disability-adjusted life-years for 32 cancer groups, 1990 to 2015: a systematic analysis for the Global Burden of Disease Study. JAMA Oncol. 2017;3(4):524-548.

4. Erdei E, Torres SM. A new understanding in the epidemiology of melanoma. Expert Rev Anticancer Ther. 2010;10(11): 1811-1823.

5. Senaratna CV, Perret JL, Lodge CJ, et al. Prevalence of obstructive sleep apnea in the general population: a systematic review. Sleep Med Rev. 2017;34:70-81.

6. Lévy P, Kohler M, McNicholas W, et al. Obstructive sleep apnoea syndrome. Nat Rev Dis Primers. 2015;1:15015.

7. McDaid C, Duree KH, Griffin SC, et al. A systematic review of continuous positive airway pressure for obstructive sleep apnoea-hypopnoea syndrome. Sleep Med Rev. 2009;13(6):427-436.

8. Campos-Rodriguez F, MartínezGarcía MA, Martinez M, et al. Association between obstructive sleep apnea and cancer incidence in a large multicenter Spanish cohort. Am J Respir Crit Care Med. 2013;187(1):99-105.

9. Martínez-García MA, CamposRodriguez F, Durán-Cantolla J, et al; Spanish Sleep Network. Obstructive sleep apnea is associated with cancer mortality in younger patients. Sleep Med. 2014;15(7):742-748.

10. Nieto FJ, Peppard PE, Young T, Finn L, Hla KM, Farré R. Sleep-disordered breathing and cancer mortality: results from the Wisconsin Sleep Cohort Study. Am J Respir Crit Care Med. 2012;186(2): 190-194.

11. Almendros I, Montserrat JM, Ramirez J, et al. Intermittent hypoxia enhances cancer progression in a mouse model of sleep apnoea. Eur Respir J. 2012;39(1): 215-217.

12. Gozal D, Farré R, Nieto FJ. Obstructive sleep apnea and cancer: epidemiologic links and theoretical biological constructs. Sleep Med Rev. 2015;27:43-55.

13. Kendzerska T, Leung RS, Hawker G, Tomlinson G, Gershon AS. Obstructive sleep apnea and the prevalence and incidence of cancer. CMAJ. 2014;186(13): 985-992.

14. Christensen AS, Clark A, Salo P, et al. Symptoms of sleep-disordered breathing and risk of cancer: a prospective cohort study. Sleep. 2013;36(10):1429-1435.

15. Martínez-García MA, CamposRodríguez F, Barbé F. Cancer and obstructive sleep apnea: current evidence from human studies. Chest. 2016;150(2): 451-463.

16. Dummer D, Hauschild A, Lindenblatt N, et al. Cutaneous melanoma: ESMO clinical practice guidelines for diagnosis, treatment and follow-up. Ann Oncol. 2015;26(suppl 5):v126-v132.

17. Nagore E, Monteagudo C, Pinazo MI, et al. [Protocol proposal for the histological report of the primary tumor in patients with cutaneous melanoma from the Task Force for Cutaneous Melanoma of the Valencian Community] [article in Spanish]. Actas Dermosifiliogr. 2007;98(7):459-465.

18. Edge SB, Compton CC. The American Joint Committee on Cancer: the 7th edition of the AJCC cancer staging manual and the future of TNM. Ann Surg Oncol. 2010;17(6):1471-1474.

19. Lloberes P, Durán-Cantolla J, MartínezGarcía MA, et al. Diagnosis and treatment of sleep apnea-hypopnea syndrome. Arch Bronconeumol. 2010;47(3):143-156.

20. Ríos L, Nagore E, López JL, et al. Melanoma characteristics at diagnosis from the Spanish National Cutaneous Melanoma Registry: 15 years of experience. Actas Dermosifiliogr. 2013;104(9):789-799. 
21. Martínez-García MA, MartorellCalatayud A, Nagore E, et al. Association between sleep disordered breathing and aggressiveness markers of malignant cutaneous melanoma. Eur Respir J. 2014;43(6):1661-1668.

22. Gozal D, Ham SA, Mokhlesi B. Sleep apnea and cancer: analysis of a nationwide population simple. Sleep. 2016;39(8):14931500.

23. Marshall NS, Wong KKH, Cullen SRJ, Knuiman MW, Grunstein RR. Sleep apnea and 20-year follow-up for all-cause mortality, stroke, and cancer incidence and mortality in the Busselton health study cohort. J Clin Sleep Med. 2014;10(4): 355-362.

24. Hakim F, Wang Y, Zhang SX, et al. Fragmented sleep accelerates tumor growth and progression through recruitment of tumor-associated macrophages and TLR4 signaling. Cancer Res. 2014;74(5):1329-1337.

25. Leibovici J, Itzhaki O, Kaptzan T, et al. Designing ageing conditions in tumour microenvironment: a new possible modality for cancer treatment. Mech Ageing Dev. 2009;130(1-2):76-85.

26. Ciocan D, Barbe C, Aubin F, et al. Distinctive features of melanoma and its management in elderly patients: a population-based study in France. JAMA Dermatol. 2013;149(10):1150-1157.

27. Rees MJ, Liao H, Spillane J, et al. Localized melanoma in older patients, the impact of increasing age and comorbid medical conditions. Eur J Surg Oncol. 2016;42(9): 1359-1366.
28. Donin N, Sinai J, Michowitz M, Hiss J, Nordenberg J, Leibovici J. Role of immune response as determinant of tumor progression in function of host age in the B16 melanoma. Mech Ageing Dev. 1995;80(2):121-137.

29. Kakizaki M, Kuriyama S, Sone T, et al. Sleep duration and the risk of breast cancer: the Ohsaki Cohort Study. Br J Cancer. 2008;99(9):1502-1505.

30. Verkasalo PK. Sleep duration and breast cancer: a prospective cohort study. Cancer Res. 2005;65(20): 9595-9600.

31. Zhang X, Giovannucci EL, Wu K, et al. Associations of self-reported sleep duration and snoring with colorectal cancer risk in men and women. Sleep. 2013;36(5):681-688. 